\title{
An eighth-order exponentially fitted two-step hybrid method of explicit type for solving orbital and oscillatory problems
}

\author{
J.M. Franco, L. Rández \\ IUMA, Departamento de Matemática Aplicada, Pza. San Francisco s/n., Universidad de \\ Zaragoza, 50009 Zaragoza, Spain.
}

\begin{abstract}
The construction of an eighth-order exponentially fitted (EF) two-step hybrid method for the numerical integration of oscillatory second-order initial value problems (IVPs) is considered. The EF two-step hybrid methods integrate exactly differential systems whose solutions can be expressed as linear combinations of exponential or trigonometric functions and have variable coefficients depending on the frequency of each problem. Based on the order conditions and the EF conditions for this class of methods, we construct an explicit EF two-step hybrid method with symmetric nodes and algebraic order eight which only uses seven function evaluations per step. This new method has the highest algebraic order we know for the case of explicit EF two-step hybrid methods. The numerical experiments carried out with several orbital and oscillatory problems show that the new eighth-order EF scheme is more efficient than other standard and EF two-step hybrid codes recently proposed in the scientific literature.
\end{abstract}

Keywords: Orbital problems; Oscillatory differential systems; Exponential fitting; Explicit two-step hybrid methods.

\section{Introduction}

Orbital problems and oscillatory differential systems often arise in different fields of applied sciences and engineering such as celestial mechanics, astronomy and astrophysics, quantum chemistry, electronics, molecular dynamics and so on (see [1, 2]), and they can be modeled by second order initial value problems (IVPs) of the form

$$
y^{\prime \prime}(t)=f(t, y(t)), \quad y\left(t_{0}\right)=y_{0}, \quad y^{\prime}\left(t_{0}\right)=y_{0}^{\prime},
$$

in which the first derivative does not appear explicitly. The numerical integration of these problems has been considered in the scientific literature by using different approaches, such as general purpose numerical methods (they have constant coefficients) or codes specially adapted to the oscillatory behavior of their solutions (they have variable coefficients depending on the frequency of each problem). The design and construction of methods with variable coefficients has been considered by several authors (see [3-28] and references therein) with the aim of using the available information on the solutions of the corresponding problems to derive more accurate and/or efficient algorithms than the general purpose algorithms for such a

*e-mail: jmfranco@unizar.es

†e-mail: randez@unizar.es 
type of problems. The most representative examples of such algorithms are the exponentially or trigonometrically fitted methods (EF or TF methods) [9-28]. After the earlier works of Gautschi [12] and others [9, 11, 13, 19], there is a well established theory on EF linear multistep methods and EF Runge-Kutta (RK)-type methods for first and second order differential systems. A detailed survey including an extensive bibliography on this subject can be found in Ixaru and Vanden Berghe [28].

Usually, the construction of EF methods consists of selecting the coefficients of the method so that it integrates exactly a set of linearly independent functions which are chosen depending on the nature of the solutions of the differential system to be solved. Several authors $[9,10,13$, $15,16,19]$ have derived EFRK methods with frequency-dependent coefficients that are able to integrate exactly first or second order differential systems whose solutions belong to the linear space generated by the set of functions $\left\{1, t, \ldots, t^{k}, \exp ( \pm \lambda t), t \exp ( \pm \lambda t), \ldots, t^{p} \exp ( \pm \lambda t)\right\}$, where $\lambda$ is a prescribed frequency. In particular, the construction of explicit EFRK-Nyström methods has been considered in $[11,17,22]$, and methods up to order five have been derived. Very recently, some authors [26, 27], have investigated the construction of explicit EF twostep hybrid methods of high order as an alternative to EFRKN methods, and they have derived methods up to order seven. It is expected that these methods integrate oscillatory problems more accurately than standard methods based on polynomial functions. In practical applications, it has been shown that EF methods are more accurate and efficient than nonfitted ones provided that the main frequency of the problem or a good approximation of it is known in advance. Therefore, the problem of how to choose a good approximation of the fitted frequency is crucial for an efficient implementation of these methods. Some procedures for the frequency determination in EF methods have been analyzed in [20, 21], but this problem is very difficult and it is still pending to be solved. Recently, Ramos and Vigo-Aguiar [24] have shown that the fitted frequency strongly depends on several factors: the differential equation, the initial conditions and the step-size.

Here, we consider the construction of explicit EF two-step hybrid methods with algebraic order eight. These methods integrate exactly second-order differential systems whose solutions can be expressed as linear combinations of the set of functions $\{\exp (\lambda t), \exp (-\lambda t)\}, \lambda \in \mathbb{C}$, or equivalently $\{\sin (\omega t), \cos (\omega t)\}$ when $\lambda=i \omega, \omega \in \mathbb{R}$. One of the most important properties for an explicit method to perform efficiently is the accuracy versus the computational cost. In general, this fact depends on the algebraic order and the number of stages (function evaluations) per step used by the method. So, the purpose of this paper is the design and construction of an eighth-order explicit EF two-step hybrid method so that the ratio number of stages/algebraic order is as small as possible, which will lead to obtain an efficient code. The paper is organized as follows: Section 2 is devoted to present the basic concepts on EF two-step hybrid methods (EF conditions, algebraic order, stability) as well as the notation to be used in the rest of the paper. In section 3 we derive an explicit EF two-step hybrid method with symmetric nodes and algebraic order eight which only requires seven function evaluations per step. This new explicit EF two-step hybrid method has the highest algebraic order we know in the literature. The stability properties of the new method are also analyzed. In section 4 we present some numerical experiments with several orbital and oscillatory IVPs that show the efficiency of the new EF two-step hybrid method when it is compared with other EF and standard two-step hybrid methods of high order proposed in the scientific literature for solving this class of problems. Section 5 is devoted to present some conclusions. 


\section{EF two-step hybrid methods}

In this section we present the EF two-step hybrid methods which are the aim of our study as well as the notation to be used in the rest of the paper. We consider $s$-stage two-step hybrid methods for solving the IVP (1), defined by the equations

$$
\begin{aligned}
Y_{i} & =\left(1+c_{i}\right) y_{n}-c_{i} y_{n-1}+h^{2} \sum_{j=1}^{s} a_{i j} f\left(t_{n}+c_{j} h, Y_{j}\right), \quad i=1, \ldots, s \\
y_{n+1} & =2 y_{n}-y_{n-1}+h^{2} \sum_{i=1}^{s} b_{i} f\left(t_{n}+c_{i} h, Y_{i}\right),
\end{aligned}
$$

where $y_{n-1}, y_{n}$ and $y_{n+1}$ represent approximations for $y\left(t_{n}-h\right), y\left(t_{n}\right)$ and $y\left(t_{n}+h\right)$, respectively. The equations (2) will be referred to as the internal stages, and equation (3) as the advance formula of the two-step hybrid method. These methods are characterized by the real parameters $b_{i}, c_{i}$ and $a_{i j}$, and they can be represented in Butcher notation by the table

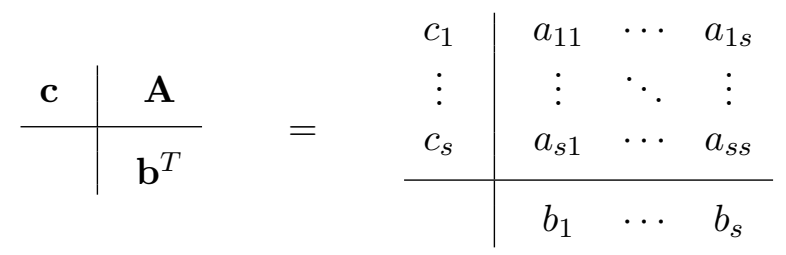

or equivalently by the triplet $(\mathbf{c}, \mathbf{A}, \mathbf{b})$.

The idea of constructing two-step hybrid methods (2)-(3) which integrate exactly a set of linearly independent functions different of the polynomials consists of selecting the available parameters in order to make the method exact for a linear space of functions with basis

$$
\mathcal{F}=\left\langle u_{1}(t), u_{2}(t), \ldots, u_{r}(t)\right\rangle, \quad r \leq s .
$$

In such case, the coefficients of the two-step hybrid method are determined by the solution of the following linear systems (see [26]):

$$
\begin{aligned}
& \sum_{i=1}^{s} b_{i} u_{k}^{\prime \prime}\left(t_{n}+c_{i} h\right)=\frac{u_{k}\left(t_{n}+h\right)-2 u_{k}\left(t_{n}\right)+u_{k}\left(t_{n}-h\right)}{h^{2}}, \quad k=1, \ldots, r \\
& \sum_{j=1}^{s} a_{i j} u_{k}^{\prime \prime}\left(t_{n}+c_{j} h\right)=\frac{u_{k}\left(t_{0}+c_{i} h\right)-\left(1+c_{i}\right) u_{k}\left(t_{n}\right)+c_{i} u_{k}\left(t_{n}-h\right)}{h^{2}} \\
& \quad i=1, \ldots, s, \quad k=1, \ldots, r .
\end{aligned}
$$

We note that the structure of the algorithm (2)-(3) indicates that it integrates exactly the solutions of the ODE $y^{\prime \prime}=0$ at the point $t_{n+1}$ of the two-step interval, irrespective of what are the coefficients. Therefore, a two-step hybrid method (2)-(3) satisfiying conditions (5)(6) is exact for the reference set of functions $\left\{1, t, u_{1}(t), u_{2}(t), \ldots, u_{r}(t)\right\}$. When $\mathcal{F}$ contains only polynomial functions up to a certain degree $\left(u_{k}(t)=t^{k+1}\right)$ the coresponding methods are the standard two-step hybrid collocation methods. If $\mathcal{F}$ contains polynomial functions and exponential or trigonometric functions, the resulting two-step hybrid methods are called mixed collocation methods or EF methods.

The most usual case is to consider exponential or trigonometric functions as reference set of functions:

$$
\mathcal{F}_{1}=\{\exp (\lambda t), \exp (-\lambda t)\} \quad \text { or } \quad \mathcal{F}_{2}=\{\sin (\omega t), \cos (\omega t)\} .
$$


The trigonometric case $\mathcal{F}_{2}$ is obtained from $\mathcal{F}_{1}$ with $\lambda=i \omega$. For the reference set of functions $\mathcal{F}_{1}$ the linear systems $(5)-(6)$ reduce to

$$
\begin{aligned}
& \sum_{i=1}^{s} b_{i} \cosh \left(c_{i} z\right)=\frac{2(\cosh (z)-1)}{z^{2}}, \quad \sum_{i=1}^{s} b_{i} \sinh \left(c_{i} z\right)=0, \\
& \sum_{j=1}^{s} a_{i j} \cosh \left(c_{j} z\right)=\frac{\cosh \left(c_{i} z\right)+c_{i} \cosh (z)-\left(1+c_{i}\right)}{z^{2}}, \quad i=1, \ldots, s, \\
& \sum_{j=1}^{s} a_{i j} \sinh \left(c_{j} z\right)=\frac{\sinh \left(c_{i} z\right)-c_{i} \sinh (z)}{z^{2}}, \quad i=1, \ldots, s,
\end{aligned}
$$

where $z=\lambda h$. The conditions defined by equations (7)-(9) characterize when a two-step hybrid method (2)-(3) is exponentially-fitted, and therefore they will be called exponential fitting conditions (EF conditions). A two-step hybrid method (2)-(3) which satisfies the EF conditions (7)-(9) will be called an EF two-step hybrid method. These EF methods integrate exactly IVPs whose solutions belong to linear spaces generated by the basis $\{1, t, \exp (\lambda t)$, $\exp (-\lambda t)\}$ or $\{1, t, \cos (\omega t), \sin (\omega t)\}$, but for IVPs with more general solutions they present local truncation errors. Therefore, an EF two-step hybrid method possesses algebraic order $p$ iff the local truncation error satisfies

$$
y\left(t_{n+1}\right)-y_{n+1}=\mathcal{O}\left(h^{p+2}\right) .
$$

In general, the local truncation error for two-step hybrid methods (2)-(3) has been analyzed by Coleman [29] by using the theory of B-series and this author has obtained an expansion of the local truncation error in the form

$$
y\left(t_{n+1}\right)-y_{n+1}=\sum_{j \geq 1} h^{j+1}\left(\sum_{\rho\left(\tau_{i}\right)=j+1} e_{j}\left(\tau_{i}\right) F\left(\tau_{i}\right)\left(y_{n}\right)\right),
$$

where $F\left(\tau_{i}\right)$ denotes the elementary differential associated to tree $\tau_{i}$, and

$$
e_{j}\left(\tau_{i}\right)=\frac{\alpha\left(\tau_{i}\right)}{(j+1) !}\left[1+(-1)^{j+1}-\mathbf{b}^{T} \boldsymbol{\Psi}^{\prime \prime}\left(\tau_{i}\right)\right], \quad \tau_{i} \in T_{2}, \quad \rho\left(\tau_{i}\right)=j+1,
$$

with $\alpha\left(\tau_{i}\right), \rho\left(\tau_{i}\right), \boldsymbol{\Psi}^{\prime \prime}\left(\tau_{i}\right)$ and $T_{2}$ defined in [29]. Therefore, having in mind that the coefficients $a_{i j}$ and $b_{i}$ of an EF two-step hybrid method are frequency-dependent and vary as functions of the step-size $h$ (they vary as functions of $z=\lambda h$ ), the necessary and sufficient conditions to reach algebraic order $p$ are given by (see Theorem 2.2 of [26])

$$
e_{\rho\left(t_{i}\right)-1}\left(t_{i}\right)=\mathcal{O}\left(z^{p+2-\rho\left(t_{i}\right)}\right), \quad \forall t_{i} \in T_{2}, \quad \text { with } \quad 2 \leq \rho\left(t_{i}\right) \leq p+1,
$$

or equivalently

$$
\mathbf{b}^{T} \boldsymbol{\Psi}^{\prime \prime}\left(t_{i}\right)=1+(-1)^{\rho\left(t_{i}\right)}+\mathcal{O}\left(z^{p+2-\rho\left(t_{i}\right)}\right), \quad \forall t_{i} \in T_{2}, \quad \text { with } \quad 2 \leq \rho\left(t_{i}\right) \leq p+1 .
$$

The $p$-th order conditions (13) or (14) up to order $p \leq 6$ are listed in [26].

The analysis of stability and phase properties is carried out by using the linear test model

$$
y^{\prime \prime}(t)=-\theta^{2} y(t), \quad \theta>0 .
$$


Since the solutions of the test model (15) have oscillatory character we will consider EF twostep hybrid methods with $\lambda=i \omega$ (the trigonometric case) where the parameter $\omega$ represents an estimate of the true frequency $\theta$ which will be used in the fitting process. When an EF two-step hybrid method of $s$ stages is applied to solve the linear test model (15), the following recursion is obtained

$$
y_{n+1}-S(H, \nu) y_{n}+P(H, \nu) y_{n-1}=0, \quad H=\theta h, \quad \nu=\omega h,
$$

where the coefficients $S(H, \nu)$ and $P(H, \nu)$ are given by

$$
\begin{aligned}
& S(H, \nu)=2-H^{2} \mathbf{b}^{T}\left(\mathbf{I}+H^{2} \mathbf{A}\right)^{-1}(\mathbf{e}+\mathbf{c}), \\
& P(H, \nu)=1-H^{2} \mathbf{b}^{T}\left(\mathbf{I}+H^{2} \mathbf{A}\right)^{-1} \mathbf{c},
\end{aligned}
$$

and the vector $\mathbf{e}=(1, \ldots, 1)^{T} \in \mathbb{R}^{s}$. If the EF method under consideration is explicit $\left(\mathbf{A}^{s-1}=0\right)$ then the coefficients $S(H, \nu)$ and $P(H, \nu)$ reduce to

$$
\begin{aligned}
& S(H, \nu)=2-\mathbf{b}^{T}(\mathbf{e}+\mathbf{c}) H^{2}+\mathbf{b}^{T} \mathbf{A}(\mathbf{e}+\mathbf{c}) H^{4}-\cdots+(-1)^{s-1} \mathbf{b}^{T} \mathbf{A}^{s-2}(\mathbf{e}+\mathbf{c}) H^{2 s-2}, \\
& P(H, \nu)=1-\left(\mathbf{b}^{T} \mathbf{c}\right) H^{2}+\left(\mathbf{b}^{T} \mathbf{A} \mathbf{c}\right) H^{4}-\cdots+(-1)^{s-1}\left(\mathbf{b}^{T} \mathbf{A}^{s-2} \mathbf{c}\right) H^{2 s-2},
\end{aligned}
$$

The stability and phase properties of the methods are determined by the roots of the characteristic polynomial of the recursion (16):

$$
\xi^{2}-S(H, \nu) \xi+P(H, \nu) .
$$

If the roots of (19) satisfy $\left|\xi_{1}(H, \nu)\right|<1$ and $\left|\xi_{2}(H, \nu)\right|<1$ there is no amplification of the error in the numerical solution and the method shows a stable behavior. If the roots of (19) are conjugate complex $\left(S(H, \nu)^{2}<4 P(H, \nu)\right)$ and satisfy $\left|\xi_{1,2}(H, \nu)\right|=1$, then the numerical solution defined by the recursion (16) is periodic as the exact solution of the linear test model (15). Otherwise, the method shows an unstable behavior.

The conditions $\left|\xi_{1}(H, \nu)\right|<1$ and $\left|\xi_{2}(H, \nu)\right|<1$ are equivalent to

$$
P(H, \nu)<1 \text { and }|S(H, \nu)|<1+P(H, \nu) .
$$

Analogously, the conditions $\left|\xi_{1,2}(H, \nu)\right|=1$ and $S(H, \nu)^{2}<4 P(H, \nu)$ are equivalent to

$$
P(H, \nu)=1 \quad \text { and } \quad|S(H, \nu)|<2 .
$$

Since the coefficients $S(H, \nu)$ and $P(H, \nu)$ of the characteristic polynomial (19) depend on $H=\theta h$ and $\nu=\omega h$, the periodicity and stability intervals typical in the classical two-step hybrid methods become two-dimensional regions in the $(H, \nu)$-plane. In order to clarify the meaning of these two-dimensional stability regions we introduce the parameter

$$
\varepsilon=\frac{\omega-\theta}{\theta},
$$

which represents the relative error in estimating the true frequency $\theta$ by the fitting frequency $\omega$. Then we have the relation $\omega=(1+\varepsilon) \theta$ or $\nu=(1+\varepsilon) H$, and the coefficients of the characteristic polynomial $(19) S(H, \varepsilon)$ and $P(H, \varepsilon)$ can be expressed in terms of the variables $H$ and $\varepsilon$. So, for EF two-step hybrid methods we use the following stability concepts:

- $R_{s}=\{(H, \varepsilon) \mid H>0, P(H, \varepsilon)<1$ and $|S(H, \varepsilon)|<1+P(H, \varepsilon)\}$ is the stability region. 
- $R_{p}=\{(H, \varepsilon) \mid H>0, P(H, \varepsilon)=1$ and $|S(H, \varepsilon)|<2\}$ is the periodicity region.

- If $R_{s}=(0, \infty) \times(-\infty, \infty)$, the method is A-stable.

- If $R_{p}=(0, \infty) \times(-\infty, \infty)$, the method is P-stable.

In addition, the quantities

$$
\phi(H, \varepsilon)=H-\arccos \left(\frac{S(H, \varepsilon)}{2 \sqrt{P(H, \varepsilon)}}\right), \quad d(H, \varepsilon)=1-\sqrt{P(H, \varepsilon)},
$$

are called the dispersion error and the dissipation error, respectively. If these quantities satisfy

$$
\phi(H, \varepsilon)=\mathcal{O}\left(\varepsilon H^{q+1}\right), \quad d(H, \varepsilon)=\mathcal{O}\left(\varepsilon H^{r+1}\right),
$$

then the method is said to be dispersive of order $q$ and dissipative of order $r$, respectively.

We note that for each value of $\varepsilon$ the stability interval is the horizontal segment inside the stability region $R_{s}$ and the periodicity interval is the horizontal segment inside the periodicity region $R_{p}$. For example, for $\varepsilon=-1(\nu=0)$ we obtain the periodicity and stability intervals of the corresponding classical two-step hybrid methods, and for $\varepsilon=0(\nu=H)$ we obtain the periodicity and stability intervals of the fitted methods in the case where the true frequency $\theta$ is known. In addition, a necessary condition for the existence of a nonempty periodicity region is $P(H, \varepsilon)=1$ as occurs in the case of symmetric or symplectic Runge-Kutta-Nyström methods.

\section{Explicit EF two-step hybrid methods of order eight}

In this section we analyze the construction of explicit EF two-step hybrid methods of order eight with the help of the order conditions and the EF conditions from the previous section. The construction of such methods is carried out by paying special attention to optimize the number of function evaluations required in each step. So, we consider the class of explicit two-step hybrid methods presented in [30]

$$
\begin{aligned}
Y_{1} & =y_{n-1}, \quad Y_{2}=y_{n}, \\
Y_{i} & =\left(1+c_{i}\right) y_{n}-c_{i} y_{n-1}+h^{2} \sum_{j=1}^{i-1} a_{i j} f\left(t_{n}+c_{j} h, Y_{j}\right), \quad i=3, \ldots, s \\
y_{n+1} & =2 y_{n}-y_{n-1}+h^{2}\left[b_{1} f_{n-1}+b_{2} f_{n}+\sum_{i=3}^{s} b_{i} f\left(t_{n}+c_{i} h, Y_{i}\right)\right],
\end{aligned}
$$

where $f_{n-1}$ and $f_{n}$ represent $f\left(t_{n-1}, y_{n-1}\right)$ and $f\left(t_{n}, y_{n}\right)$, respectively, the two first nodes are $c_{1}=-1, c_{2}=0$, and which can be represented by the table of coefficients

$$
\begin{array}{c|cc|ccccc} 
& & -1 & 0 & 0 & 0 & \cdots & 0 \\
0 & 0 & 0 & 0 & \cdots & 0 \\
c & A \\
\hline & b^{T} & c_{31} & a_{32} & 0 & \cdots & 0 \\
\vdots & \vdots & \vdots & \ddots & \ddots & \vdots \\
c_{s} & a_{s 1} & a_{s 2} & \cdots & a_{s, s-1} & 0 \\
\hline & b_{1} & b_{2} & \cdots & b_{s-1} & b_{s}
\end{array}
$$


These methods (after the starting procedure) only require $s-1$ function evaluations in each step, and therefore they can be considered as two-step hybrid methods with $s-1$ stages per step. In addition, we consider the case of methods with symmetric nodes and weights. These symmetry conditions produce a significant simplification on the EF conditions (7) and on the order conditions (14), which make easier the determination of the EF methods. For example, these conditions imply that the second equation in the EF conditions (7) is satisfied as well as the order conditions $\mathbf{b}^{T} \mathbf{c}^{2 j-1}=0, j \geq 1$. Examples of an explicit EF two-step hybrid method with symmetric nodes and weights up to order seven are derived in [26].

\subsection{Explicit EF two-step hybrid methods with $s=8$}

In order to obtain eighth-order efficient methods we consider $s=8$ so that the ratio number of stages/algebraic order is small. In addition, we consider the following simplifying assumptions:

$$
b_{3}=0, \quad \sum_{j \geq 1} a_{i j} c_{j}^{2}=\frac{c_{i}^{4}+c_{i}}{12}+\mathcal{O}\left(z^{2}\right), \quad i=4, \ldots, s
$$

which give rise to a significant simplification of the eighth-order conditions. So, we analyze the case of explicit two-step hybrid methods with symmetric nodes and weights which are defined by the table of coefficients

\begin{tabular}{c|cccccccc}
-1 & 0 & 0 & 0 & 0 & 0 & 0 & 0 & 0 \\
0 & 0 & 0 & 0 & 0 & 0 & 0 & 0 & 0 \\
$c_{3}$ & $a_{31}$ & $a_{32}$ & 0 & 0 & 0 & 0 & 0 & 0 \\
$c_{4}$ & $a_{41}$ & $a_{42}$ & $a_{43}$ & 0 & 0 & 0 & 0 & 0 \\
$-c_{4}$ & $a_{51}$ & $a_{52}$ & $a_{53}$ & $a_{54}$ & 0 & 0 & 0 & 0 \\
$-c_{3}$ & $a_{61}$ & $a_{62}$ & $a_{63}$ & $a_{64}$ & $a_{65}$ & 0 & 0 & 0 \\
$c_{3}$ & $a_{71}$ & $a_{72}$ & $a_{73}$ & $a_{74}$ & $a_{75}$ & $a_{76}$ & 0 & 0 \\
1 & $a_{81}$ & $a_{82}$ & $a_{83}$ & $a_{84}$ & $a_{85}$ & $a_{86}$ & $a_{87}$ & 0 \\
\hline & $b_{1}$ & $b_{2}$ & 0 & $b_{4}$ & $b_{4}$ & $b_{6}$ & $b_{6}$ & $b_{1}$
\end{tabular}

In order to determine the weights we impose that the advance formula is also exact for the reference set of functions $\left\{t^{2}, t^{3}, t^{4}, t^{5}, t^{6}, t^{7}\right\}$, in addition to EF conditions (7), and these conditions reduce to

$$
\mathbf{b}^{T} \cosh (\mathbf{c} z)=\frac{2(\cosh (z)-1)}{z^{2}}, \quad \mathbf{b}^{T} \mathbf{e}=1, \quad \mathbf{b}^{T} \mathbf{c}^{2}=\frac{1}{6}, \quad \mathbf{b}^{T} \mathbf{c}^{4}=\frac{1}{15},
$$

where

$$
\cosh (\mathbf{c} z)=\left(\cosh \left(c_{1} z\right), \ldots, \cosh \left(c_{s} z\right)\right)^{T} .
$$

Solving equations (29), the weights $b_{1}, b_{2}, b_{4}$ and $b_{6}$ may be determined in terms of the arbitrary parameters $c_{3}$ and $c_{4}$ and the variable $z$.

In order to determine the coefficients $a_{i j}$ we impose the EF conditions (8)-(9), the order conditions (14) with $p=8$ (algebraic order eight) and the simplifying assumptions (28). The solution of these equations gives rise to the fact that the coefficients $a_{i j}$ (with $j \geq 3$ ) may be considered independent of $z$ and they can be determined in terms of the arbitrary parameters $c_{3}, c_{4}$ and $a_{84}$. In particular, the coefficients $a_{i 1}$ and $a_{i 2}(3 \leq i \leq 8)$ may be computed from the EF conditions (8)-(9) in terms of the arbitrary parameters $c_{3}, c_{4}, a_{84}$ and the variable $z$, 
the coefficients $a_{i 3}(4 \leq i \leq 8)$ may be computed from the simplifying assumptions $(28)$ and the rest of coefficients may be computed from the eighth-order conditions (14). Consequently, these coefficients define a three-parameter family of eighth-order explicit EF two-step hybrid methods which require seven function evaluations per step.

Now, we select the free parameters $c_{3}, c_{4}$ and $a_{84}$ so that the constants of the dispersion and dissipation errors at $z=0(\varepsilon=-1)$ are small, obtaining the values

$$
\begin{gathered}
c_{3}=-\frac{3}{5}, \quad c_{4}=-\frac{1}{5}, \quad a_{84}=0, \\
\phi(H,-1)=\frac{36991 H^{9}}{410780160000}+\mathcal{O}\left(H^{11}\right)=9.005 \times 10^{-8} H^{9}+\mathcal{O}\left(H^{11}\right), \\
d(H,-1)=\frac{2580331 H^{10}}{17515464300000}+\mathcal{O}\left(H^{12}\right)=1.47 \times 10^{-7} H^{10}+\mathcal{O}\left(H^{12}\right),
\end{gathered}
$$

and the remaining coefficients are given by

$$
\begin{aligned}
& b_{1}=\frac{25 z^{2} \cosh \left(\frac{z}{5}\right)-25 z^{2} \cosh \left(\frac{3 z}{5}\right)-48\left(2+z^{2}-2 \cosh (z)\right)}{32 z^{2}\left(-128+150 \cosh \left(\frac{z}{5}\right)-25 \cosh \left(\frac{3 z}{5}\right)+3 \cosh (z)\right)}, \\
& b_{2}=\frac{768+250 z^{2} \cosh \left(\frac{z}{5}\right)+125 z^{2} \cosh \left(\frac{3 z}{5}\right)-768 \cosh (z)+9 z^{2} \cosh (z)}{3 z^{2}\left(-128+150 \cosh \left(\frac{z}{5}\right)-25 \cosh \left(\frac{3 z}{5}\right)+3 \cosh (z)\right)}, \\
& b_{4}=-\frac{25\left(32\left(18+5 z^{2}\right)+125 z^{2} \cosh \left(\frac{3 z}{5}\right)+3\left(-192+z^{2}\right) \cosh (z)\right)}{96 z^{2}\left(-128+150 \cosh \left(\frac{z}{5}\right)-25 \cosh \left(\frac{3 z}{5}\right)+3 \cosh (z)\right)}, \\
& b_{6}=\frac{25\left(96-80 z^{2}+125 z^{2} \cosh \left(\frac{z}{5}\right)+3\left(-32+z^{2}\right) \cosh (z)\right)}{96 z^{2}\left(-128+150 \cosh \left(\frac{z}{5}\right)-25 \cosh \left(\frac{3 z}{5}\right)+3 \cosh (z)\right)}, \\
& a_{43}=-\frac{29}{450}, \quad a_{53}=\frac{61}{900}, \quad a_{54}=-\frac{1}{150}, \quad a_{63}=-\frac{52}{1415}, \\
& a_{64}=\frac{13717}{21225}, \quad a_{65}=\frac{4849}{12735}, \quad a_{73}=\frac{1079}{42450}, \quad a_{74}=-\frac{9886}{21225}, \\
& a_{75}=-\frac{13453}{50940}, \quad a_{76}=\frac{233}{11320}, \quad a_{83}=\frac{805}{5409}, \quad a_{85}=\frac{23915}{21636}, \\
& a_{86}=\frac{2045}{43272}, \quad a_{87}=\frac{2440}{5409}, \\
& a_{31}=\frac{5 \sinh \left(\frac{3 z}{5}\right)-3 \sinh (z)}{5 z^{2} \sinh (z)}, \quad a_{32}=\frac{5 \cosh \left(\frac{3 z}{5}\right)-5 \operatorname{coth}(z) \sinh \left(\frac{3 z}{5}\right)-2}{5 z^{2}},
\end{aligned}
$$




$$
\begin{aligned}
& a_{41}=\frac{5 \sinh \left(\frac{z}{5}\right)-\sinh (z)+\frac{29}{90} z^{2} \sinh \left(\frac{3 z}{5}\right)}{5 z^{2} \sinh (z)}, \\
& a_{42}=\frac{450 \cosh \left(\frac{z}{5}\right)+29 z^{2} \cosh \left(\frac{3 z}{5}\right)-\left(450 \sinh \left(\frac{z}{5}\right)+29 z^{2} \sinh \left(\frac{3 z}{5}\right)\right) \operatorname{coth}(z)-360}{450 z^{2}}, \\
& a_{51}=\frac{6\left(z^{2}-150\right) \sinh \left(\frac{z}{5}\right)-61 z^{2} \sinh \left(\frac{3 z}{5}\right)+180 \sinh (z)}{900 z^{2} \sinh (z)}, \\
& a_{52}=\frac{6\left(150+z^{2}\right) \cosh \left(\frac{z}{5}\right)+\left(900+55 z^{2}+122 z^{2} \cosh \left(\frac{2 z}{5}\right)\right) \operatorname{coth}(z) \sinh \left(\frac{z}{5}\right)-61 z^{2} \cosh \left(\frac{3 z}{5}\right)-1080}{900 z^{2}}, \\
& a_{61}=\frac{38205 \sinh (z)-16906 z^{2} \sinh \left(\frac{z}{5}\right)+45\left(52 z^{2}-1415\right) \sinh \left(\frac{3 z}{5}\right)}{63675 z^{2} \sinh (z)}, \\
& a_{62}=\frac{45\left(1415+52 z^{2}\right) \cosh \left(\frac{3 z}{5}\right)+\operatorname{coth}(z)\left(16906 z^{2} \sinh \left(\frac{z}{5}\right)+45\left(1415-52 z^{2}\right) \sinh \left(\frac{3 z}{5}\right)\right)}{63675 z^{2}} \\
& -\frac{101880+65396 z^{2} \cosh \left(\frac{z}{5}\right)}{63675 z^{2}} \\
& a_{71}=\frac{\frac{51367 z^{2} \sinh \left(\frac{z}{5}\right)}{50940}+\left(5-\frac{821 z^{2}}{33960}\right) \sinh \left(\frac{3 z}{5}\right)-3 \sinh (z)}{5 z^{2} \sinh (z)}, \\
& a_{72}=\frac{\left(509400-23433 z^{2}\right) \cosh \left(\frac{3 z}{5}\right)-203760+371794 z^{2} \cosh \left(\frac{z}{5}\right)}{509400 z^{2}} \\
& +\frac{\left(2463 z^{2} \sinh \left(\frac{3 z}{5}\right)-102734 z^{2} \sinh \left(\frac{z}{5}\right)-509400 \sinh \left(\frac{3 z}{5}\right)\right) \operatorname{coth}(z)}{509400 z^{2}}, \\
& a_{81}=\frac{23915\left(2 \sinh \left(\frac{z}{5}\right)-\sinh \left(\frac{3 z}{5}\right)\right)}{43272 \sinh (z)}, \\
& a_{82}=-\frac{23915 \operatorname{coth}(z)\left(2 \sinh \left(\frac{z}{5}\right)-\sinh \left(\frac{3 z}{5}\right)+47830 \cosh \left(\frac{z}{5}\right)+28005 \cosh \left(\frac{3 z}{5}\right)\right)}{43272} \\
& +\frac{86544(\cosh (z)-1)}{43272 z^{2}} .
\end{aligned}
$$

The new eighth-order explicit EF two-step hybrid method will be denoted as EFTSHM8 and it has the highest algebraic order that can be found in the literature for this type of EF methods.

We note that the advance formula of the new method EFTSHM8 is exact for the basis $\left\langle 1, t, t^{2}, t^{3}, t^{4}, t^{5}, t^{6}, t^{7}, \exp (\lambda t), \exp (-\lambda t)\right\rangle$ or $\left\langle 1, t, t^{2}, t^{3}, t^{4}, t^{5}, t^{6}, t^{7}, \cos (\omega t), \sin (\omega t)\right\rangle$. The coefficients for the trigonometric case $(\lambda=i \omega, \omega \in \mathbb{R})$ emerge having in mind the relations $\cosh (i \nu)=\cos (\nu)$ and $\sinh (i \nu)=i \sin (\nu)$, where $\nu=\omega h$. In addition, for small values of $|z|$ the formulas for the coefficients of the method are subject to heavy cancellations, and when $|z|<0.1$, series expansions for the coefficients must be used. 


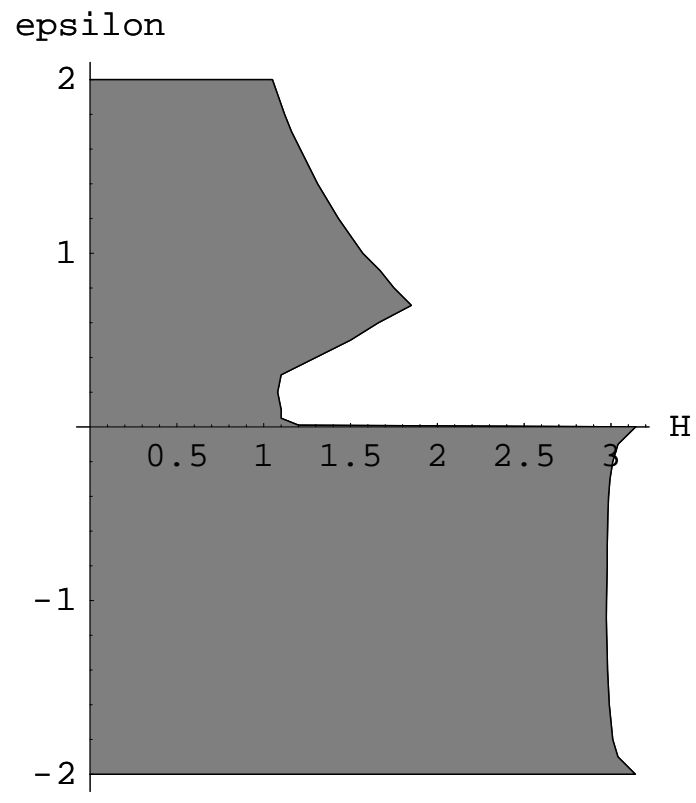

Figure 1. Primary stability region for the new scheme EFTSHM8.

We also note that another way of expressing the coefficients of the method EFTSHM8 is to use the functions $\xi(Z), \eta_{0}(Z), \eta_{1}(Z), \ldots$, introduced by Ixaru in [31], and extensively used in [28]. This fact remove the vulnerability of the coefficients of the method when $|z|$ is small, and the procedure for doing this is shown in the Appendix.

\subsection{Stability of the new eighth-order method}

Finally, we analyze the stability of the new eighth-order EF two-step hybrid method derived above. We note that the new scheme is dissipative $(P(H, \varepsilon)<1)$ and therefore it has a nonempty stability region. In addition, the range of interest to analyze the stability properties of fitted methods is $|\varepsilon| \leq 1$. If the relative error satisfies $|\varepsilon|>1$, the estimate of the true frequency $\theta$ is very bad and then it is preferable to use a classical method (case $\varepsilon=-1$ ). Figure 1 shows the primary stability region for the new EF method. This Figure shows that for the case of $\varepsilon=-1$ (classical methods) the classical counterpart of scheme EFTSHM8 has stability interval $(0,2.97)$.

\section{Numerical experiments}

In this section we present some numerical experiments to test the numerical efficiency of the new eighth-order explicit EF two-step hybrid integrator derived in section 3 when it is applied to the numerical solution of several orbital problems and related oscillatory IVPs. The new EF integrator EFTSHM8 has been compared with the following standard and EF schemes denoted by:

- ETSHM7TF: The seventh-order TF two-step hybrid method derived by of Kalogiratou et al. [27]. 
- EFTSHM7: The seventh-order EF two-step hybrid method derived in [26].

- TSHM81: The eighth-order standard two-step hybrid method derived by Tsitouras [32].

- TSHM8 ${ }_{2}$ : The eighth-order standard two-step hybrid method derived by Famelis [33].

The criterion used in the numerical comparisons is the usual test based on computing the maximum global error in the solution $\left(\mathrm{MGE}=\log _{10}\left(\max \left\|y\left(t_{n}\right)-y_{n}\right\|\right)\right)$ over the whole integration interval, and the numerical tests were carried out on a PC Intel Pentium computer. The algorithms were implemented in python by using the mpmath library with a precision of thirty two significant digits. Figures $2-5$ show the maximum global error in the solution (MGE) versus the computational cost measured by the number of $f$-evaluations (in logarithmic scale) required by each method.

Problem 1. We consider the two-body gravitational problem (Kepler's plane problem) defined by the IVP

$$
\begin{aligned}
& q_{1}^{\prime \prime}=-\frac{q_{1}}{\left(q_{1}^{2}+q_{2}^{2}\right)^{3 / 2}}, \quad q_{1}(0)=1-e, \quad q_{1}^{\prime}(0)=0 \\
& q_{2}^{\prime \prime}=-\frac{q_{2}}{\left(q_{1}^{2}+q_{2}^{2}\right)^{3 / 2}}, \quad q_{2}(0)=0, \quad q_{2}^{\prime}(0)=\sqrt{\frac{1+e}{1-e}}
\end{aligned}
$$

where $e(0 \leq e<1)$ represents the eccentricity of the orbit and whose exact solution is a $2 \pi$-periodic elliptic orbit with semimajor axis 1 given by

$$
q_{1}(t)=\cos (u(t))-e, \quad q_{2}(t)=\sqrt{1-e^{2}} \sin (u(t)),
$$

where $u(t)$ is the solution of Kepler's equation: $t=u(t)-e \sin (u(t))$. The integration is carried out on the interval $[0,200 \pi]$ with fitting parameter $\omega=1(\lambda=i \omega)$, and we select the eccentricity values $e=0.05$ and $e=0.25$. The numerical results obtained for this problem are presented in Figures 2a and 2b.

Problem 2. We consider a perturbed Kepler's problem defined by the IVP

$$
\begin{array}{ll}
q_{1}^{\prime \prime}=-\frac{q_{1}}{\left(q_{1}^{2}+q_{2}^{2}\right)^{3 / 2}}-\delta \frac{(2+\delta) q_{1}}{\left(q_{1}^{2}+q_{2}^{2}\right)^{5 / 2}}, \quad q_{1}(0)=1, & q_{1}^{\prime}(0)=0, \\
q_{2}^{\prime \prime}=-\frac{q_{2}}{\left(q_{1}^{2}+q_{2}^{2}\right)^{3 / 2}}-\delta \frac{(2+\delta) q_{2}}{\left(q_{1}^{2}+q_{2}^{2}\right)^{5 / 2}}, \quad q_{2}(0)=0, & q_{2}^{\prime}(0)=1+\delta
\end{array}
$$

where $\delta$ is a small positive parameter and whose analytic solution is

$$
q_{1}(t)=\cos (t+\delta t), \quad q_{2}(t)=\sin (t+\delta t) .
$$

The numerical results presented in Figure 3 have been computed with fitting parameter $\omega=1(\lambda=i \omega)$, parameter of the perturbation $\delta=10^{-2}$, and the problem is integrated up to $t_{\text {end }}=400$.

Problem 3. We consider the two-dimensional semi-linear oscillatory second-order IVP

$$
q^{\prime \prime}(t)=-\frac{1}{2}\left(\begin{array}{cc}
\alpha & \beta \\
\beta & \alpha
\end{array}\right) q(t)+g(q(t)), \quad q(0)=\left(\begin{array}{c}
1 / 2 \\
1 / 2
\end{array}\right), \quad q^{\prime}(0)=\left(\begin{array}{c}
-1 / \sqrt{2}-\omega / 2 \\
1 / \sqrt{2}-\omega / 2
\end{array}\right)
$$


where

$$
g(q(t))=\frac{k^{2}}{2}\left(q_{1}(t)-q_{2}(t)\right)^{3}\left(\begin{array}{c}
1 \\
-1
\end{array}\right), \quad \alpha=\omega^{2}+k^{2}+1, \quad \beta=\omega^{2}-k^{2}-1,
$$

and the parameters $\omega>0,0 \leq k<1$.

This oscillatory IVP represents a simple model consisting of two mass points connected with a soft nonlinear spring and a stiff linear spring, and its analytic solution represents a periodic motion in terms of trigonometric and Jacobi's elliptic functions given by

$$
q(t)=\frac{1}{\sqrt{2}}\left(\begin{array}{c}
\cos \left(\frac{\pi}{4}+\omega t\right)-\operatorname{sn}(t ; k) \\
\cos \left(\frac{\pi}{4}+\omega t\right)+\operatorname{sn}(t ; k)
\end{array}\right) .
$$

In our test we choose the parameter values $\omega=50, k=0.1, t_{\text {end }}=100$, and the numerical results are presented in Figure 4.

Problem 4. We consider the popular Bessel equation [33]

$$
q^{\prime \prime}(t)=\left(-100+\frac{1}{4 t^{2}}\right) q(t), \quad q(1)=J_{0}(10), \quad q^{\prime}(1)=-0.5576953439142885,
$$

where $J_{0}$ is the Bessel function of the first kind of order zero and whose analytic solution is

$$
q(t)=\sqrt{t} J_{0}(10 t) .
$$

This oscillatory IVP is solved until finding the 100th root of the solution which occurs at $t=32.59406213134967$, and the numerical results are presented in Figure 5.

From the numerical results obtained in Figures 2-5 it follows that for the orbital problems and oscillatory IVPs under consideration the new explicit EF two-step hybrid integrator of order eight (EFTSHM8) shows a more efficient behavior than the other integrators used in the comparison when high accuracy is required. Except in the case of Problem 1 with $e=0.05$, the new integrator EFTSHM8 turns out to be the most efficient of all tested codes when low or high accuracy is required. This is due to the fact that the scheme EFTSHM8 has a larger accuracy order than the fitted schemes ETSHM7TF and EFTSHM7 and the same accuracy order than the standard schemes TSHM $8_{1}$ and $\mathrm{TSHM}_{2}$. In the case of orbital problems (Problems 1 and 2) the fitted integrator ETSHM7TF turns out to be slightly more efficient than EFTSHM7. However, for highly oscillatory systems (Problem 3) the scheme ETSHM7TF gives the poorest results. Finally, we can observe (Figures 4 and 5) that for oscillatory IVPs in which the linear terms are dominant (Problems 3 and 4) the eighth-order standard methods TSHM8 $8_{1}$ and TSHM8 $8_{2}$ specially designed for solving oscillatory problems may be more efficient than seventh-order fitted methods ETSHM7TF and EFTSHM7 when very high accuracy is required.

\section{Conclusions}

A study on the construction of eighth-order explicit EF two-step hybrid methods for solving orbital problems and second-order oscillatory IVPs has been carried out. This study is based on combining the EF conditions, the order conditions and a reduced number of stages (low 


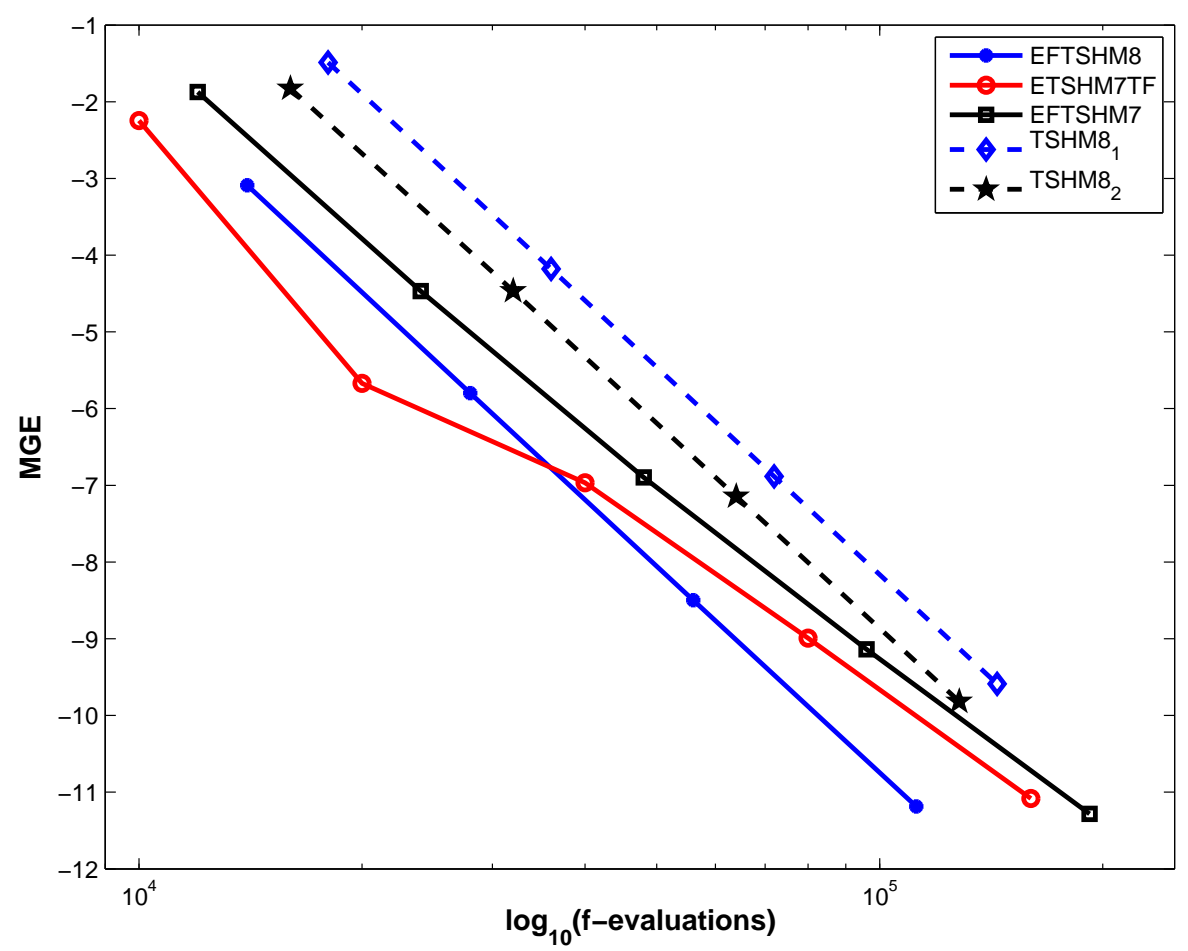

Figure 2a. Efficiency curves for Problem 1 with $e=0.05$.

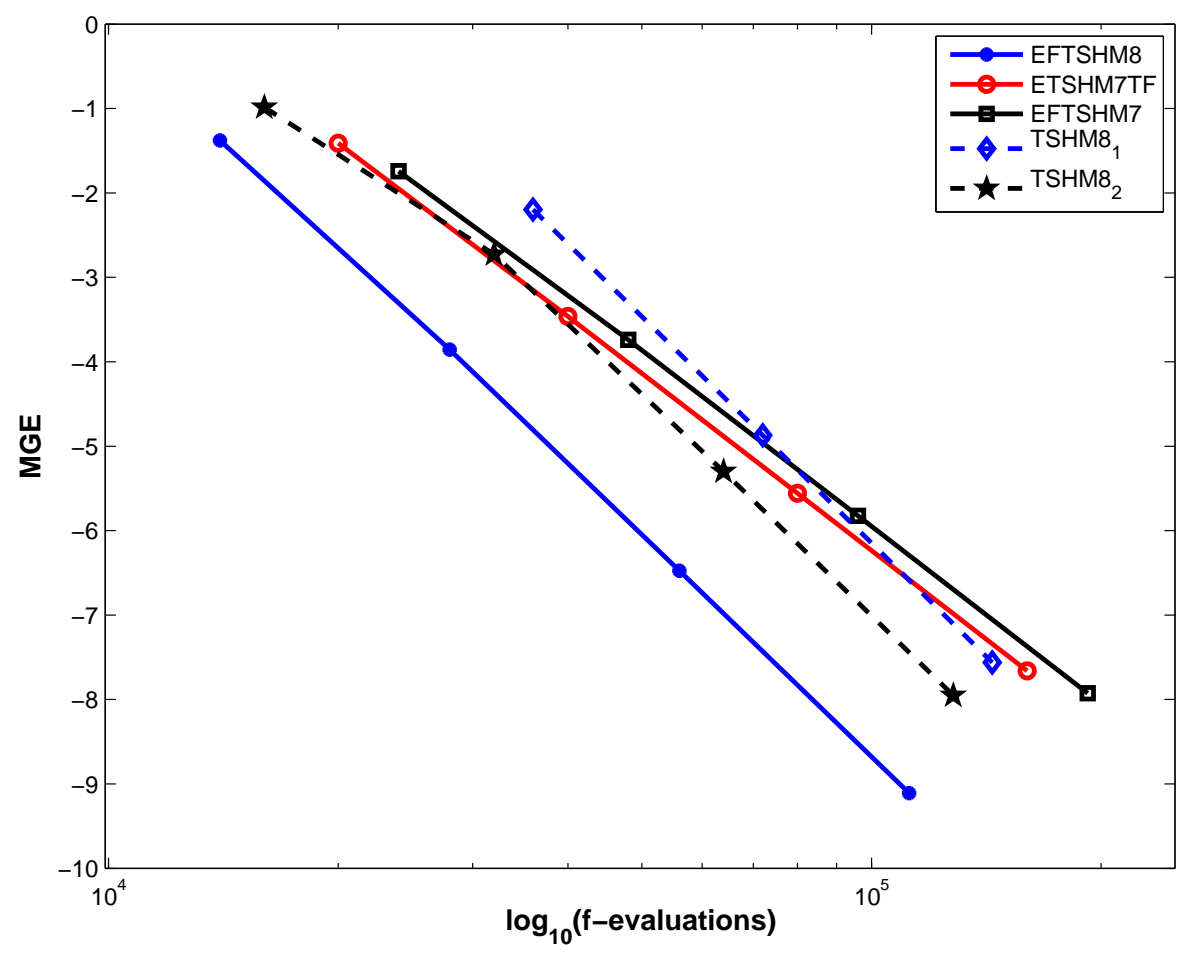

Figure 2b. Efficiency curves for Problem 1 with $e=0.25$. 


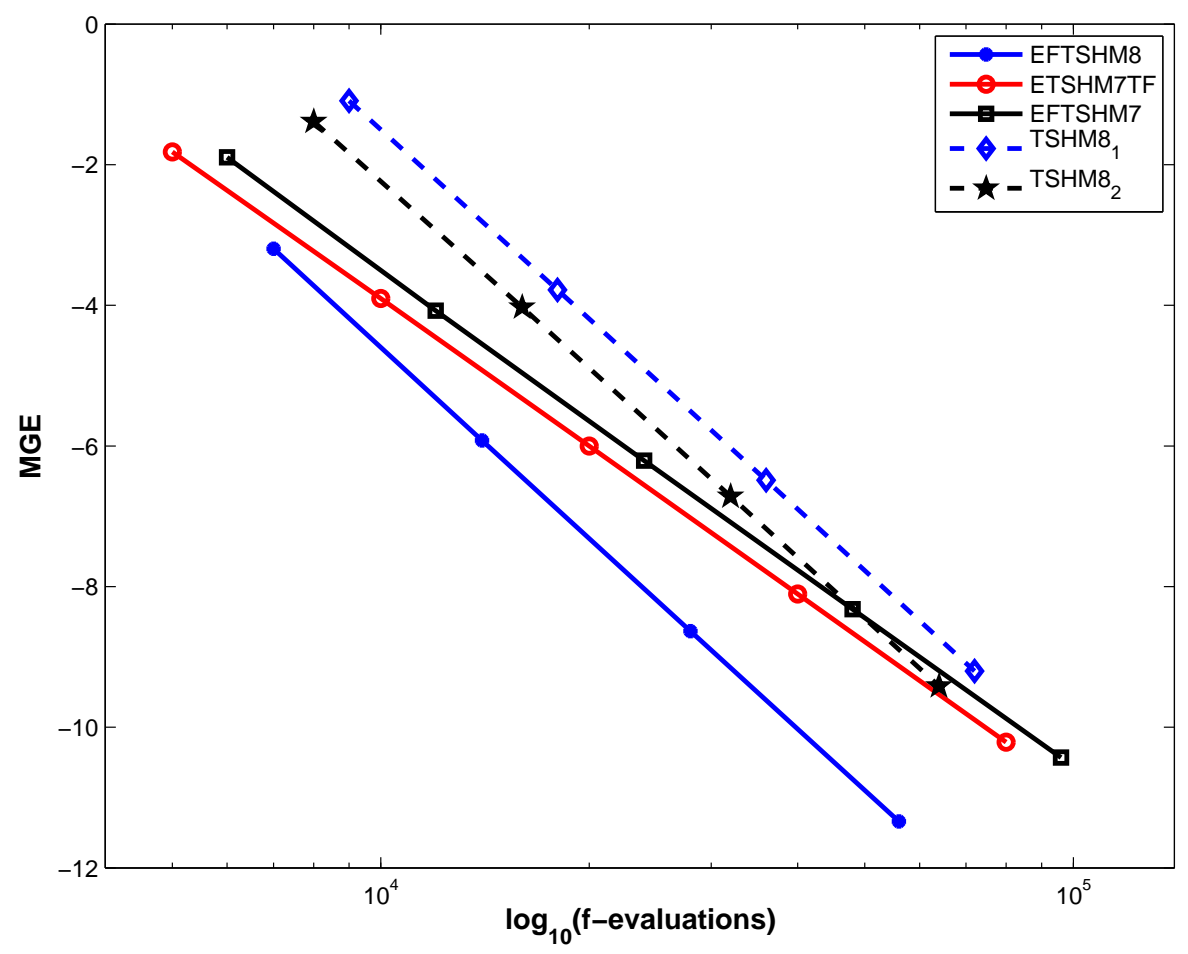

Figure 3. Efficiency curves for Problem 2.

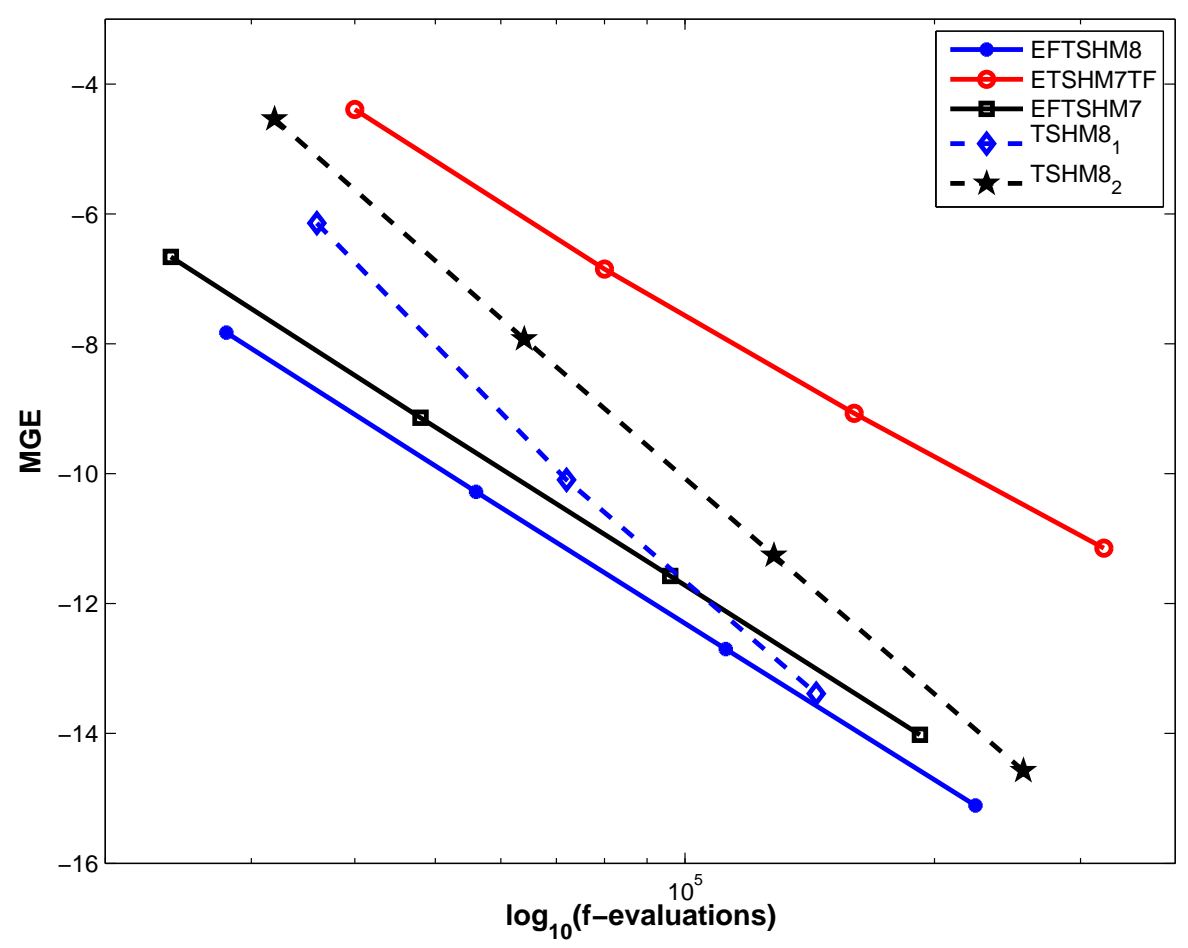

Figure 4. Efficiency curves for Problem 3. 


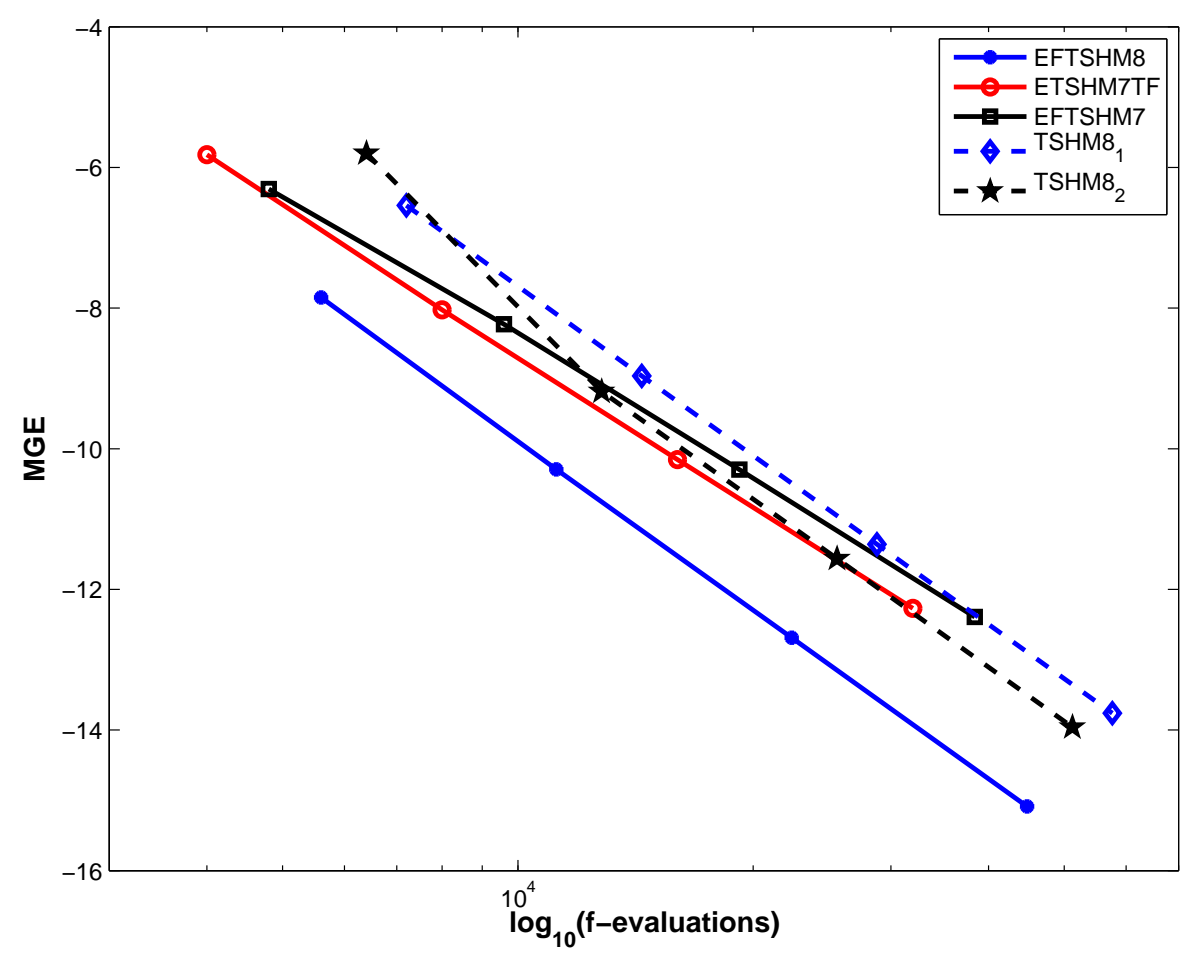

Figure 5. Efficiency curves for Problem 4.

cost) in order to obtain efficient methods. A new eighth-order explicit EF two-step hybrid scheme which is not an EF version of a standard two-step hybrid method from the literature is constructed. This new explicit EF two-step integrator shows to be a reliable alternative to high-order standard $[32,33]$ and fitted $[26,27]$ two-step hybrid methods specially designed for solving oscillatory problems. The numerical experiments carried out with several orbital problems and related oscillatory IVPs show that the new eighth-order explicit EF two-step integrator improves the computational efficiency obtained with standard and fitted two-step hybrid methods of high order from the scientific literature.

\section{Appendix}

In order to avoid the vulnerability of the coefficients of the method EFTSHM8 when $|z|$ is small, the special functions introduced by Ixaru in [31] which are extensively used in [28] can be used.

So, we start with Ixaru function $\xi(Z)$,

$$
\xi(Z)= \begin{cases}\cos \left(|Z|^{1 / 2}\right), & \text { if } Z \leq 0 \\ \cosh \left(|Z|^{1 / 2}\right), & \text { if } Z>0\end{cases}
$$

(in [28] this is denoted $\eta_{-1}(Z)$ ). Notice that this covers both trigonometric and hyperbolic cases depending on the sign of $\mathrm{Z}$ but, instead of $\eta_{0}(Z), \eta_{1}(Z), \ldots$, we introduce the following 
set of functions:

$$
u_{0}(Z)=(\xi(Z)-1) / Z, \quad u_{i}(Z)=\left(u_{i-1}(Z)-1 /(2 i) !\right) / Z, \quad i \geq 1,
$$

with the following properties:

i) $u_{i}(0)=1 /[2(i+1)] !, \quad i \geq 0$.

ii) Series expansions: $u_{i}(Z)=\sum_{k=i+1}^{\infty} Z^{k-i-1} /(2 k) !, \quad i \geq 0$.

iii) Reverse relations: $\xi(Z)=Z u_{0}(Z)+1, \quad u_{i}(Z)=Z u_{i+1}(Z)+1 /[2(i+1)]$ !, $\quad i \geq 0$.

For an accurate computation of these functions you can fix a threshold $(\delta)$ and use the definition formulas for $|Z|>\delta$ and the truncated series for $|Z|<\delta$ (just over five terms are perhaps sufficient in most computations).

Now we focus our attention on the coefficient $b_{1}$ on page 8 and express its denominator and numerator in terms of these functions. We will see that a new factor $\mathrm{Z}$ appears as we advance with $\mathrm{i}$. The chain is stopped when the value at $\mathrm{Z}=0$ of the multiplying factor is nonvanishing.

Denominator of $b_{1}$ :

$$
\begin{aligned}
\text { Dem } & =32 z^{2}\left(-128+150 \cosh \left(\frac{z}{5}\right)-25 \cosh \left(\frac{3 z}{5}\right)+3 \cosh (z)\right) \\
& =32 Z[-128+150 \xi(Z / 25)-25 \xi(9 Z / 25)+3 \xi(Z)] \\
& * \quad \text { Use } u_{0}(Z) \quad\left(\xi(Z)=Z u_{0}(Z)+1\right) \\
& =96 Z^{2}\left[2 u_{0}(Z / 25)-3 u_{0}(9 Z / 25)+u_{0}(Z)\right]
\end{aligned}
$$

* The expression in the square brackets is zero at $Z=0$ then we go on with $u_{1}(Z)$ $\left(u_{0}(Z)=Z u_{1}(Z)+1 / 2\right.$ !)

$=\frac{96}{25} Z^{3}\left[2 u_{1}(Z / 25)-27 u_{1}(9 Z / 25)+25 u_{1}(Z)\right]$

* The expression in the square brackets is zero at $Z=0$ then we go on with $u_{2}(Z)$ $\left(u_{1}(Z)=Z u_{2}(Z)+1 / 4 !\right)$

$=\frac{96}{625} Z^{4}\left[2 u_{2}(Z / 25)-243 u_{2}(9 Z / 25)+625 u_{2}(Z)\right]$

* The process is now stopped because the value of the expression in the square brackets at $Z=0$ is nonvanishing.

Numerator of $b_{1}$ : On applying the same technique we get

$$
\begin{aligned}
N u m & =25 z^{2} \cosh \left(\frac{z}{5}\right)-25 z^{2} \cosh \left(\frac{3 z}{5}\right)-48\left(2+z^{2}-2 \cosh (z)\right) \\
& =25 Z \xi(Z / 25)-25 Z \xi(9 Z / 25)-48(2+Z-2 \xi(Z)) \\
& =Z^{2}\left[u_{0}(Z / 25)-9 u_{0}(9 Z / 25)+96 u_{1}(Z)\right]
\end{aligned}
$$




$$
\begin{aligned}
& =\frac{1}{25} Z^{3}\left[u_{1}(Z / 25)-81 u_{1}(9 Z / 25)+2400 u_{2}(Z)\right] \\
& =\frac{1}{625} Z^{4}\left[u_{2}(Z / 25)-729 u_{2}(9 Z / 25)+60000 u_{3}(Z)\right]
\end{aligned}
$$

Finally, we obtain the coefficient

$$
b_{1}=\frac{u_{2}(Z / 25)-729 u_{2}(9 Z / 25)+60000 u_{3}(Z)}{96\left[2 u_{2}(Z / 25)-243 u_{2}(9 Z / 25)+625 u_{2}(Z)\right]},
$$

whose computation is uniformly accurate irrespective of the values of the positive/negative $Z$, or, equivalently, for real/imaginary $\lambda$. In a similar way, the rest of the coefficients of the method EFTSHM8 can be expresed in terms of the functions $u_{i}(Z) . i \geq 0$.

\section{Acknowledgements}

The authors are grateful to the anonymous reviewers for their valuable suggestions, which help to improve the paper. This research has been partially supported by Project MTM201347318-C2-1-P of the Dirección General de Investigación (Ministerio de Educación, Cultura y Deporte). We are grateful to J.J. Martínez for helping us in preparing the manuscript.

\section{References}

[1] L.D. Landau and F.M. Lifshitz, Quantum Mechanics, Pergamon Press, New York, 1965.

[2] R.L. Liboff, Introductory Quantum Mechanics, Addison-Wesley, Reading, MA, 1980.

[3] D.G. Bettis, Runge-Kutta algorithms for oscillatory problems, J. Appl. Math. Phys. (ZAMP), 30 (1979) 699-704.

[4] A.B. González, P. Martín and J.M. Farto, A new family of Runge-Kutta type methods for the numerical integration of perturbed oscillators, Numer. Math., 82 (1999) 635-646.

[5] J.M. Franco, Runge-Kutta-Nyström methods adapted to the numerical integration of perturbed oscillators, Comput. Phys. Comm., 147 (2002) 770-787.

[6] H. Yang, X. Wu, X. You and Y. Fang, Extended RKN-type methods for numerical integration of perturbed oscillators, Comput. Phys. Comm., 180 (2009) 1777-1794.

[7] H. Van de Vyver, An explicit Numerov-type method for second-order differential equations with oscillating solutions, Comput. Math. Appl., 53 (2007) 1339-1348.

[8] D.F. Papadopoulos, Z.A. Anastassi, T.E. Simos, A phase-fitted Runge-Kutta-Nyström method for the numerical solution of initial value problems with oscillating solutions, Comput. Phys. Comm., 180 (2009) 1839-1846.

[9] J.P. Coleman and S.C. Duxbury, Mixed collocation methods for $y^{\prime \prime}=f(x, y)$, J. Comput. Appl. Math., 126 (2000) 47-75.

[10] J.M. Franco, An embedded pair of exponentially fitted explicit Runge-Kutta methods, J. Comput. Appl. Math., 149 (2002) 407-414. 
[11] J.M. Franco, Exponentially fitted explicit Runge-Kutta-Nyström methods, J. Comput. Appl. Math., 167 (2004) 1-19.

[12] W. Gautschi, Numerical integration of ordinary differential equations based on trigonometric polynomials, Numer. Math. 3 (1961) 381-397.

[13] K. Ozawa, A functional fitting Runge-Kutta method with variable coefficients, Japan J. Indust. Appl. Math., 18 (2001) 107-130.

[14] K. Ozawa, A functionally fitted three-stage explicit singly diagonally implicit RungeKutta method, Japan J. Indust. Appl. Math., 22 (2005) 403-427.

[15] B. Paternoster, Runge-Kutta(-Nyström) methods for ODEs with periodic solutions based on trigonometric polynomials, Appl. Numer. Math., 28 (1998) 401-412.

[16] T.E. Simos, An exponentially-fitted Runge-Kutta method for the numerical integration of initial-value problems with periodic or oscillating solutions, Comput. Phys. Commun., 115 (1998) 1-8.

[17] T.E. Simos, Exponentially-fitted Runge-Kutta-Nyström method for the numerical solution of initial-value problems with oscillating solutions, Appl. Math. Lett., 15 (2002) $217-225$.

[18] G. Vanden Berghe, H. De Meyer, M. Van Daele and T. Van Hecke, Exponentially-fitted explicit Runge-Kutta methods, Comput. Phys. Commun., 123 (1999) 7-15.

[19] G. Vanden Berghe, H. De Meyer, M. Van Daele and T. Van Hecke, Exponentially fitted Runge-Kutta methods, J. Comput. Appl. Math., 125 (2000) 107-115.

[20] G. Vanden Berghe, L. Gr. Ixaru, H. De Meyer: Frequency determination and steplength control for exponentially fitted Runge-Kutta methods, J. Comput. Appl. Math., 132 (2001), pp. 95-105.

[21] L. Gr. Ixaru, G. Vanden Berghe, H. De Meyer: Frequency evaluation in exponential fitting algorithms for ODEs, J. Comput. Appl. Math., 140 (2002), 423-434.

[22] H. Van de Vyver, An embedded exponentially fitted Runge-Kutta-Nyström method for the numerical solution of orbital problems, New Astronomy, 11 (2006) 577-587.

[23] Y. Fang, Y. Song and X. Wu, Trigonometrically fitted explicit Numerov-type method for periodic IVPs with two frequencies, Comput. Phys. Commun., 179 (2008) 801-811.

[24] H. Ramos and J. Vigo-Aguiar: On the frequency choice in trigonometrically fitted methods, Appl. Math. Lett., 23 (2010) 1378-1381.

[25] R. D'Ambrosio, E. Esposito, B. Paternoster, Exponentially fitted two-step hybrid methods for $y^{\prime \prime}=f(x, y)$, J. Comput. Appl. Math., 235 (2011) 4888-4897.

[26] J.M. Franco, L. Rández, Explicit exponentially fitted two-step hybrid methods of high order for second-order oscillatory IVPs, Appl. Math. Comput., 273 (2016) 493-505.

[27] Z. Kalogiratou, Th. Monovasilis, Higinio Ramos, T.E. Simos, A new approach on the construction of trigonometrically fitted two step hybrid methods, J. Comput. Appl. Math., 303 (2016) 146-155. 
[28] L. Gr. Ixaru and G. Vanden Berghe, Exponential Fitting, Kluwer Academic Publishers, 2004.

[29] J.P. Coleman, Order conditions for a class of two-step methods for $y^{\prime \prime}=f(x, y), I M A J$. Numer. Anal., 23 (2003) 197-220.

[30] J.M. Franco, A class of explicit two-step hybrid methods for second-order IVPs, $J$. Comput. Appl. Math., 187 (2006) 41-57.

[31] L. Gr. Ixaru, Operations on oscillatory functions, Comput. Phys. Commun., 105 (1997) $1-19$.

[32] Ch. Tsitouras, Explicit eighth order two-step methods with nine stages for integrating oscillatory problems, Int. J. Mod. Phys. C, 17 (2006) 861-876.

[33] I. Th. Famelis, Explicit eighth order Numerov-type methods with reduced number of stages for oscillatory IVPs, Int. J. Mod. Phys. C, 19 (2008) 957-970. 